\title{
Comparison of Online Adaptive Learning Algorithms for Myoelectric Hand Control
}

\author{
Yue Zhang \\ College of Computer Science and Technology \\ Zhejiang University of Technology \\ Hangzhou, China PR \\ pagesiyang@gmail.com
}

\author{
Yinfeng Fang and Honghai Liu \\ School of Computing \\ University of Portsmouth \\ Portsmouth, United Kingdom \\ yinfeng.fang@port.ac.uk, honghai.liu@port.ac.uk
}

\begin{abstract}
Pattern recognition (PR) based myoelectric hand control has become a research focus in the field of rehabilitative engineer and intelligent control. However, the state of the art method is hardly adopted for clinical use because of signal interfered by shift, fatigue and user-unfriendly of retraining. The aim of this study is to evaluate the performance of different kinds of online algorithms in classifying the myoelectric hand motions, and reveal the key factors to classification accuracy of online learning algorithms. Two groups of experiments on intra-session and inter-session were designed to evaluate the classification and recognition performance of overall methods. The comparison results show that the second-order online learning algorithms outperformed the first-order algorithms in classification and recognition. Soft confidence-weighted learning performs best with $99 \%$ classification rate in same session and over $85 \%$ recognition rate in different session. This paper uncovers the online learning with large margin and confidence weight can always acquire a good property. In addition, online learning algorithms retrain the classification model by incorporating the testing data to the previous model by measuring the changes between the predicted label and true label which can improve the performance in long-term use.
\end{abstract}

Keywords-Surface Electromyography; Pattern Recogniton; Online Learning Algorithm; Hand Motion

\section{INTRODUCTION}

Recent years, surface electromyography (sEMG) prosthetic hand control has attracted wide attention in biomimetic engineering, which can make amputee more dexterous to control multiple DOFs prosthetic hand. Among different kind of control strategies, myoelectric control based on pattern recognition $(\mathrm{PR})$ is regarded as the optimizing approach for the moment. Because PR-based method can extract more useful information from the available muscles than conventional methods [1], and therefore, it can provide more intuitive and reliable control of prosthetic hand.

Although myoelectric pattern recognition approach is a promising method to control multifunctional dexterous prosthesis [2 3], most of the studies are implemented in a controlled laboratory environment and the data are usually collected by harsh terms in a single session. As a result, the classification error is low within session in special condition but decreased dramatically in daily life. Conventional pattern recognition methods divide the classification procedure into two parts, the first part is training step for revealing the underlying relationship from training data, the second part is testing step used to classify testing data with the model from training step. The model from training step contain limited information because the training data were collected just in a short period which cannot representative for the whole period including testing step. It is impossible to prepare adequate training data which include all information. Until now, no PRbased prosthetic systems are accepted by clinical application because of the unstable performance of these existing off-line pattern recognition algorithms in daily life settings [4] due to the changes between training data to testing data generated by muscle fatigue [5], electrode shifts [6], and arm position changes [7].

A robust myoelectric pattern recognition system can decrease the effects from physical factors listed above, and increase the classification accuracy over sessions. To decrease the time cost spent in retraining myoelectric pattern recognition system whenever classification performance changes observably, online adaptive learning algorithm has proposed in recent studies. The major focus is two kinds of adaptive training: user-training and algorithm-training. User-training is to enhance the control ability of human beings themselves. Michael et al. [8] utilizes a real-time biofeedback system with virtual prosthesis control to make classifier reliability for at least a standard workday a likely outcome for a trained user. Through this scheme, amputees can learn to produce the consistent, distinguishable muscle patterns. Hargrove et al. [9] found that slight shift from training position can make the classification accuracy sudden decrease from $90 \%$ to $60 \%$ for a 10-class myoelectric control problem. Algorithm-training is to improve the pattern recognition algorithms robust performance, and make it adjust parameters form training model by itself when external factors changes. Online adaptive learning algorithm is motivated to incrementally retrain the classifier online with the testing data by measuring the real-time changes between training data and testing data. Liu J. [10] proposed an adaptive unsupervised classifier based on support vector machine (SVM). This method takes the real-time changes between testing data and training data into consideration when predict the classification label, and then some adjustments on this changes would be added to the classification model in an unsupervised manner. Thus, continuously updating the model parameters makes the classifier adaptive to the changes. Chen 
et al. [11] extend the off-line pattern recognition methods: linear discriminant analysis (denoted as LDA for short) and quadratic discriminant analysis (denoted as QDA for short) to self-enhancing methods (SELDA and SEQDA) which continuously updating the class mean vectors, the class covariance and the pooled covariance using the testing data respectively. The classification accuracy is $2.2 \%$ and $1.6 \%$ higher than that of conventional LDA and QDA respectively in short period, and the classification accuracy of SEQDA is $3.15 \%$ better than that of QDA in long period. Nishikawa et al. [12] proposed an on-line learning mechanism. Using this mechanism, the mapping relation of surface electromyogram to motions of prosthetic hands can be obtained with the evaluation from amputees. This method adjust the parameters of model in a supervised manner. The classification rate is $9.6 \%$ higher than conventional methods. But this mechanism did not provide an evaluation criterion to decide which training data should be selected for recalculating the classifier.

Compared to conventional pattern recognition algorithms, such as $\mathrm{K}$ Nearest Neighbor (KNN), Linear Discriminant Analysis (LDA) and Support Vector Machine (SVM), online adaptive learning algorithms perform better in training step and testing step in short and long term. Batch algorithms collect a large number of labelled samples for training, it can obtain good accuracy in single session, but failed in long-term use. Once new training data need to handle, the model should be retrain with all training data, resulting in time consuming, computationally expensive and not be feasible in practice [12]. Whereas online learning algorithms do not need vast training samples, the algorithm can gain a simple model by a small quantity of samples, then the testing data comes in and the model will be updated incrementally by these new data with or without labels, which is scalable and time saving.

In this study, we will make comparisons in different online adaptive algorithms based on perceptron. A series of evaluation criteria include classification accuracy and stability will be used to measure the applicability in pattern recognition based sEMG signal for prosthetic hand. At last, a discussion will be held according to the theory of the algorithm and experimental result.

\section{MEthodS}

Many classification problems existed in real world are linearly separable or separable approximation [20]. In literature, linear algorithms have the advantages of simple construction and low computation [17]. So far, many excellent linear classification algorithms such as LDA, KNN, and Perceptron etc. have acquired great success in various fields. Based on the nature of linear algorithms, some online learning classification algorithms have been proposed in recent studies. Although different online learning algorithms exhibit different properties in classification, they share the common algorithmic framework of online learning. In general, these algorithms can be distinguished in terms of different definitions and designs of the loss function and their update functions, which might be founded on different theories and principles [17]. According to the distinction of the correction about weight vector, online learning algorithms can be generally grouped into two major categories: 1) first-order online learning algorithms [13 1416 23], and 2) second-order online learning algorithms [ll $1518 \quad 19$ 21 22]. The first-order algorithms only keep updating one classification function based on gradient decent method, which carry out when new sample import. While the second-order algorithms typically assume the weight vector follows a Gaussian distribution and then each learning step will update the mean vector and covariance matrix by the new sample. The second-order online learning algorithms can better exploit the underlying structures between features of the data which are missed by the firstorder algorithms [15].

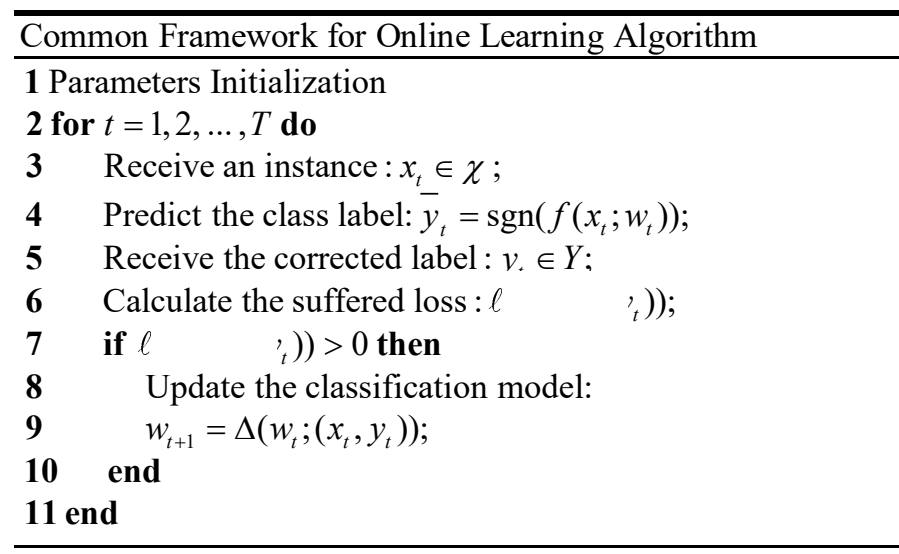

In the following section, several online learning algorithms for multiclass classification will be introduced briefly, including first-order algorithms e.g. Perceptron [pp], Online gradient descent (OGD) learning [23], Passive aggressive (PA) learning [16]. Second-order algorithms e.g. Confidenceweighted (CW) learning [18 21], Adaptive regularization of weight (AROW) learning [19], Soft confidence weighted (SCW) learning [22].

\section{A. Perceptron algorithm}

The perceptron algorithm [13] is the earliest and simplest method for online learning. The weighted vector is initialized to any value and updated step by step by gradient descent. Crammer and Singer extended the original Perceptron algorithm to multiclass Perceptron algorithm [14]. Given an input example, multiclass algorithm will compute a similarity score between each prototype and the input example, then the index of the prototype which achieved the highest similarity will be set to the predicted label. The algorithm allocates different weights on the support vectors corresponding to those error-set. The perceptron is the base of most online learning algorithms for binary and multiclass classification.

The optimization of perceptron is simplest, which can be formulated by following:

$w_{t+1}=w_{t}+y_{t} x_{t}$ 


\section{B. Online Gradient Descent algorithm}

The online gradient descent algorithm [23] exploits the gradient decent updating approach for optimizing the objective function defined by different type of loss functions.

The optimization of OGD learning algorithm can be divided into four categories, they are formulated as following:

1) When the loss_type $=0$ (0-1 loss)

$w_{t+1}=w_{t}+\eta_{t} y_{t} x_{t}$

$$
\text { s.t. } \left.\left.\quad i_{t}\right)\right)=\mathrm{I}\left(\mathrm{y}_{t} \neq \overline{y_{t}}\right)
$$

2) When the loss_type $=1$ (hinge loss)

$$
\begin{aligned}
& w_{t+1}=w_{t}+\eta_{t} y_{t} x_{t} \\
& \text { s.t. } \left.\left.\ell \quad '_{t}\right)\right)=\max \left(0,1-y_{t}\left(w_{t} \cdot x_{t}\right)\right)
\end{aligned}
$$

3) When the loss_type $=2$ (logistic loss)

$$
\begin{aligned}
& w_{t+1}=w_{t}+\eta_{t} y_{t} x_{t} * \frac{1}{1+\exp \left(y_{t}\left(w_{t} \cdot x_{t}\right)\right)} \\
& \text { s.t. } \left.\left.\ell \quad \quad_{t}\right)\right)=\log \left(1+\exp \left(-y_{t}\left(w_{t} \cdot x_{t}\right)\right)\right)
\end{aligned}
$$

4) When the loss_type $=3$ (square loss)

$$
\begin{aligned}
& w_{t+1}=w_{t}+\eta_{t}\left(y_{t}-w_{t} \cdot x_{t}\right) x_{t} \\
& \text { s.t. } \left.\left.\quad \quad{ }_{t}\right)\right)=0.5 *\left(w_{t} \cdot x_{t}-y_{t}\right)^{2}
\end{aligned}
$$

\section{Passive Aggressive algorithm}

In general, online algorithms only update the weight vector by minimize the cumulative loss of the model. Passive Aggressive algorithm [16] not only considers the above restriction, but also keep close between new weight vector and last weight vector as much as possible. This algorithm is a compromise way between passiveness (minimizing the distance between the new classifier and the previous one) and aggressiveness (minimizing the loss of the new classifier suffered on the current instance). The optimization of PA learning algorithm is formulated as following:

$$
\begin{aligned}
& w_{t+1}=\arg \min _{w \in \mathbb{R}}-\left\|_{w}-w_{t}\right\|^{2} \\
& \text { s.t. } \ell \quad))=0
\end{aligned}
$$

where the loss function is based on the hinge loss:

$$
\ell \quad)=\left\{\begin{array}{lr}
0 & \text { if } y_{t}\left(w \cdot x_{t}\right) \geq 1 \\
1-y_{t}\left(w \cdot x_{t}\right) & \text { otherwise }
\end{array}\right.
$$

The above optimization has the update rule like below:

$$
w_{t+1}=w_{t}+\eta_{t}^{P A} y_{t} x_{t} \text {, where } \eta_{t}^{P A}=\stackrel{\ell}{ }\left\|x_{t}\right\|^{2} \stackrel{\left.\left.{ }_{t}^{\prime}\right)\right)}{ }
$$

Further, to make PA have adaptation with non-separable instances and more robust, a slack variable $\varepsilon$ was introduced into the optimization. With linear and quadratic penalty, two extended methods: PA-I and PA-II are formulated as:

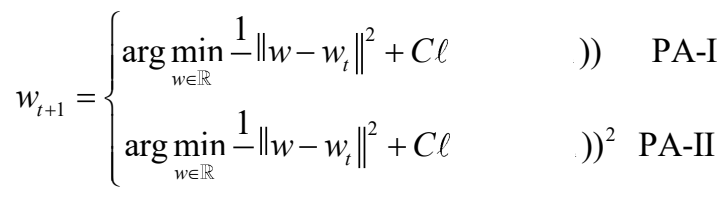

where $C$ is a parameter for tradeoff between passiveness and aggressiveness. The update rule is the same as above but different coefficients $\eta_{t}$ as follows:

$$
\eta_{t}^{\mathrm{PA}-\mathrm{I}}=\min \left\{C,{ }^{\ell} \quad\left\|x_{t}\right\|^{2} \quad,, \cdot t_{t} \quad \ell \quad\left\|x_{t}\right\|^{2}+\frac{1}{2 C}\right.
$$

\section{Confidence-Weighted learning algorithm}

Unlike the geometrical margin constraint of most online algorithms, the confidence-weighted learning [18 21] is constrained by probability and a classifier would have a high classifier accuracy with high probability. It maintains a Gaussian distributions over weight vectors with a mean vector and covariance matrix which reflect the uncertainly relation between weights and correlations. The distribution can be used to control the direction and scale of parameter updates within model. The weight distribution is updated by minimizing the Kullback-Leibler divergence between the new weight distribution and the old one while ensuring that the probability of correct classification is greater than a threshold as follows:

$$
\begin{aligned}
& \left(\mu_{t+1}, \Sigma_{t+1}\right)=\underset{\mu, \Sigma}{\arg \min _{\mu L}} D_{K L}(\mathbb{N} \quad \mathbb{N} \\
& \text { s.t. } P r_{w \sim \mathbb{N}} \quad\left[y_{t}\left(w \cdot x_{t}\right) \geq 0\right] \geq \eta
\end{aligned}
$$

CW learning can provide a precise connection between weight uncertainty and prediction confidence while computation is feasible.

The optimization problem has the update rules as follows:

$$
u_{t+1}=u_{t}+\alpha_{t} y_{t} \Sigma_{t} x_{t} \quad \Sigma_{t+1}=\Sigma_{t}-\beta_{t} \Sigma_{t} x_{t}^{\mathrm{T}} x_{t} \Sigma_{t}
$$

\section{E. Adaptive Regularization of Weight Vectors algorithm}

Adaptive regularization of weight learning algorithm [19] performs adaptive regularization of the prediction function when new instance comes up. That is to say, it would adjust its regularization for each example. AROW have several attractive properties: large margin training, confidence weighting, and capacity to handle non-separable data. It is proposed based on $\mathrm{CW}$ learning algorithm to overcome the sudden changes in the classification function due to label noise. The optimization is formulated as follows:

$$
\begin{aligned}
\left(\mu_{t+1}, \Sigma_{t+1}\right)= & \arg \min _{\mu, \Sigma} D_{K L}(\mathbb{N} \quad \mathbb{N} \\
& \left.\left.\frac{1}{2 \gamma} \ell \quad v_{t}\right)\right)+\frac{1}{2 \gamma} x_{t}^{\mathrm{T}} \Sigma_{t} x_{t}
\end{aligned}
$$

where $\left.\left.\ell \quad v_{t}\right)\right)=\left(\max \left\{1-y_{t}\left(\mu \cdot x_{t}\right)\right\}\right)^{2}$ and $\gamma$ is a regularization parameter.

The update rule of optimization is the same as (11) . 


\section{F. Online Gradient Descent algorithm}

The soft confidence weighted algorithm [22] is the upgraded version of $\mathrm{CW}$ algorithm and AROW algorithm. It extends the confidence-weighted learning for soft margin learning which makes SCW learning more robust. SCW owns four salient properties: (I) large margin training, (II) confidence weighting, (III) capability to handle non-separable data, and (IV) adaptive margin. With these properties, SCW learning can overcome label noise and gain extra efficiency and effectiveness.

The optimization of the SCW-I is formulated as follows:

$$
\begin{aligned}
\left(\mu_{t+1}, \Sigma_{t+1}\right)= & \arg \min _{\omega \sim} D_{K L}(\mathbb{N} \quad \mathbb{N} \\
& \left.\left.+C \ell \quad v_{t}\right)\right)
\end{aligned}
$$

where $C$ is a parameter to trade off the passiveness and aggressiveness.

SCW-II employs a squared penalty to previous formula.

$$
\begin{aligned}
\left(\mu_{t+1}, \Sigma_{t+1}\right)= & \arg \min _{\| \Sigma} D_{K L}(\mathbb{N} \quad \mathbb{N} \\
& \left.\left.+C \ell \quad v_{t}\right)\right)^{2}
\end{aligned}
$$

The update rule of classification rule is:

$$
\mu_{t+1}=\mu_{t}+\alpha_{t} y_{t} \Sigma_{t} x_{t}, \Sigma_{t+1}=\Sigma_{t}-\beta_{t} \Sigma_{t} x_{t}^{\mathrm{T}} x_{t} \Sigma_{t}
$$

\section{EXPERIMENT SETUP}

\section{A. Subject}

Two healthy subjects volunteered for the sEMG signal capture in the laboratory condition. [Subject 1, age: 43, mass: $72 \mathrm{~kg}$, height: $174 \mathrm{~cm}$ ][Subject 2, age: 30 , mass: $60 \mathrm{~kg}$, height: $170 \mathrm{~cm}]$. They are all right handed and no previous history of neuropathies or traumas to the upper limbs.

\section{B. Apparatus}

To obtain sEMG signals from forearm, a multi-channel sEMG acquisition system [26] is employed. This device supports up to 16 bipolar EMG channel, 5000 amplification gains and 12 bits ADC resolution. The signal is filtered by a band pass filter embedded in device from $20 \mathrm{~Hz}$ to $500 \mathrm{~Hz}$ which can remove motion artifacts and white noise, and meanwhile a notch filter with $50 \mathrm{~Hz}$ center frequency is used to filter power line noises in hardware. Finally the signal was sampled at $1 \mathrm{kHz}$ and transferred to a $\mathrm{PC}$ that installed Windows 7 operating system via USB port, and a custom designed software is used to save the sEMG signal data after removing the power-line noise and its harmonic component further by a comb filter.

We placed on each subject's dominant forearm 18 surface EMG electrodes and 16 channels EMG data were collected from forearm muscles. The ways of placement of the electrodes is Zig configuration [24], seen in Fig. 1, which is proved as the optimizing electrodes configuration and it can improve the EMG pattern discrimination. The configuration of electrodes used in this paper ignore the muscle positions.

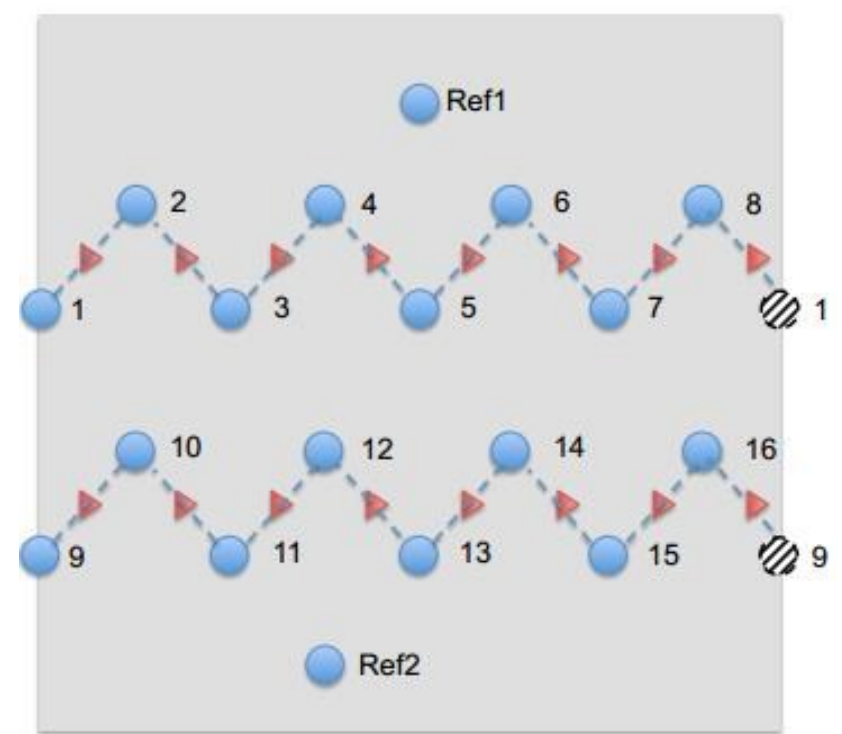

Fig. 1. Zig electrode layout. The blue solid circles labelled from 1 to 16 represent sEMG electrodes. The Red triangle indicates a bipolar sEMG channel connecting two neighbor electrodes connected by dot lines. Electrode Ref1 and Ref 2 are the reference electrodes.

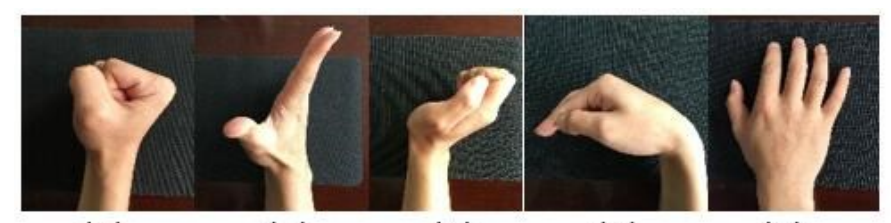

(1)

(2)

(3)

(4)

(5)

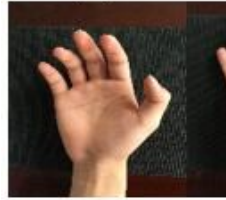

(6)

(7)

(8)

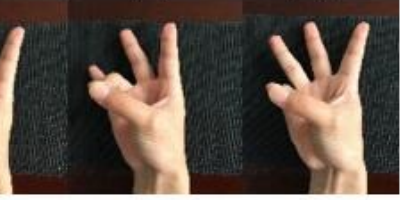

(9)

(10)

Fig. 2. Ten hand motions are used in this paper for hand recognition. 1 Hand Close, 2 Hand Open, 3 Wrist Flexion, 4 Wrist Extension, 5 Pronation, 6 Supination and 7-10 thumb opposition towards index finger, middle finger, ring finger and little finger.

\section{Data Collection}

The subject was informed to remain seated and keep the elbow fixed during capturing. Ten hand motions were considered and sEMG signal for each channel was exacted. In the experiment, we define a trail as a repetition of a hand motion, and 10 trials are repeated for each motion. A session means collecting data with the fixed position of electrode sleeve in one day. Three sessions of data collection were completed for each subject. In each session, sEMG signal data of ten motion (1 Hand Close, 2 Hand Open, 3 Wrist Flexion, 4 Wrist Extension, 5 Pronation, 6 Supination and 7-10 thumb opposition towards index finger, middle finger, ring finger and little finger) are collected in accordance with the sequence. Subjects repeat a motion with 10 times according to the cue signal at $5 \mathrm{~s}, 15 \mathrm{~s}, 25 \mathrm{~s}, \ldots 95 \mathrm{~s}$, and maintain a motion for $5 \mathrm{~s}$ until an end cue be given at $10 \mathrm{~s}, 20 \mathrm{~s}, 30 \mathrm{~s}, \ldots, 100 \mathrm{~s}$.

The placement of electrodes and sleeve was instructed to the subjects before experiments. To simplify the positioned way of electrodes, we request subjects to wear the electrode 


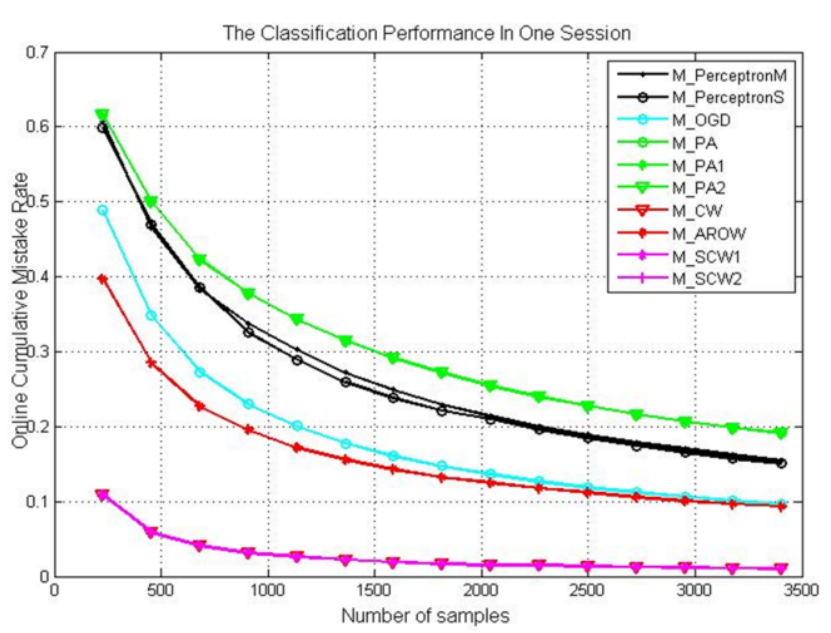

(a)

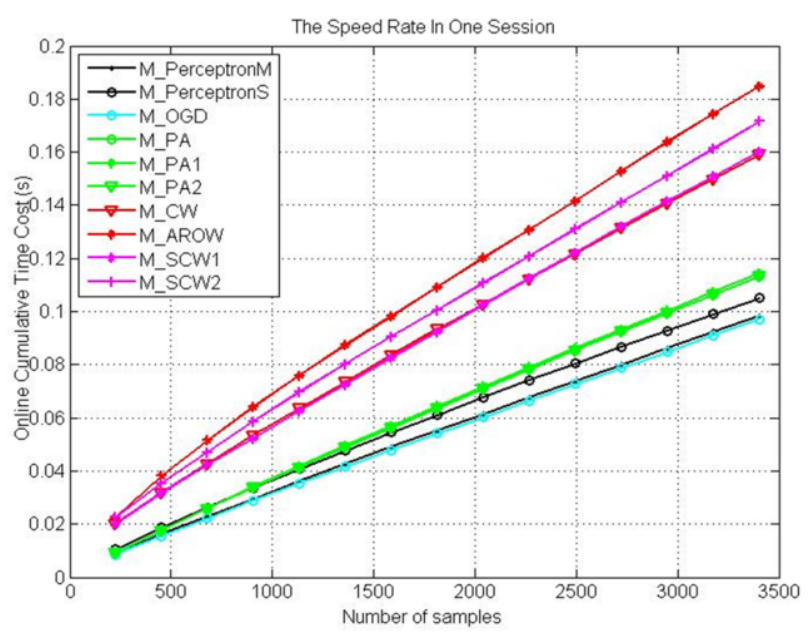

(b)

Fig. 3. Classification performance in one session. (a) show the cumulative mistake rate which is relevant to classification property of algorithm itself. (b) show the cumulative time cost which is relevant to the efficiency between different online learning algorithms.

sleeve without label and pretreatment on the skin [25], then another empty sleeve with no electrodes is used to cover the previous sleeve for the sake of generating a squeeze to every electrodes towards the skin. It is suggested to keep 10 minutes interval between different hand motion capture in order to keep the muscle fatigue at same level.

\section{Signal Processing and Pattern Recognition}

Data segment is closely relevant to systemic response time and the classification accuracy [27]. We divided the raw signal into some little segments by a sliding window scheme. Here a $300 \mathrm{~ms}$ window and $100 \mathrm{~ms}$ incremental window are adopted. A $300 \mathrm{~ms}$ window length provide adequate information and a $100 \mathrm{~ms}$ incremental window is sufficient for real-time requirement.

The combination of autoregressive (AR) model coefficients [28] and time domain (TD) feature [29] acts as the overall feature set in our study. To guarantee the classification in real time, AR2 is chose to produce two features per channel. Root mean square (RMS) [28] and waveform length (WL) [28] comprise the two TD features. There is a clear linear relationship between the features [20]. For each motion with 10 trials, only the steady-state signals were selected for classification. The transitory signals are not considered in this paper, because a prosthesis would not be able to respond to transitory signal due to mechanical [30]. 10 trails of each motion construct a single dataset. Only the steady signal in $[6 \mathrm{~s}$, $9 \mathrm{~s}],[16 \mathrm{~s}, 19 \mathrm{~s}]$, etc. were extracted.

We compared different online algorithms on collected dataset. The extra parameters e.g. parameter $r$ in AROW, parameter $C$ in PA, PA-I, PA-II, SW-I and SW-II are all determined by across validation in order to select the best one from $\left\{2^{-4}, 2^{-3}, \ldots, 2^{3}, 2^{4}\right\}$, the parameter $\eta$ in CW, SCW-I, SCW-II are determined by cross validation to select the best one from $\{0.5,0.55, \ldots, 0.9,0.95\}$. These parameters are used for Initialization of the algorithms.

\section{RESULT AND DISSCUSION.}

\section{A. Intra-session performance of the online algorithms}

To evaluate the performance of different online algorithms, same dataset within one session is used in this experiment. We always assume the dataset from different sessions are independent in this experiment because each session was executed in parallel condition. First, we choose a feature set randomly to verify which algorithm can separate the training set effective and efficient, the result can be seen in figure 3 . In the aspect of cumulative mistake rate, apparently, secondorder algorithms outperform the first-order algorithms by a large gap. Among the second-order algorithms, SCW performs the best. In the aspect of cumulative time cost, first-order algorithms get a little advantage, but the difference can be ignored in real-time system.

Only one session cannot represent the final performance of algorithms. So we select a feature set from one session, and then 20 random permutations of the set will be generated for

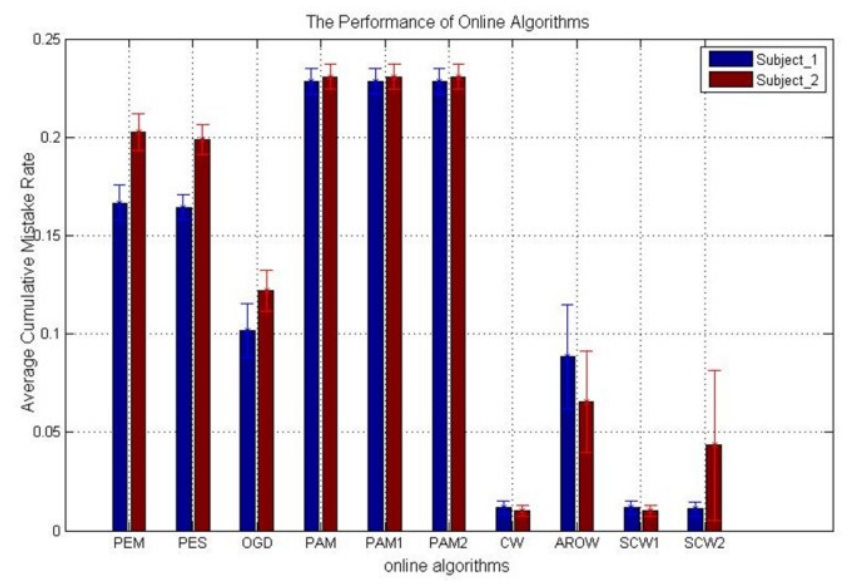

Fig. 4. The average cumulative mistake rate and deviation of classification performance between different sessions. 


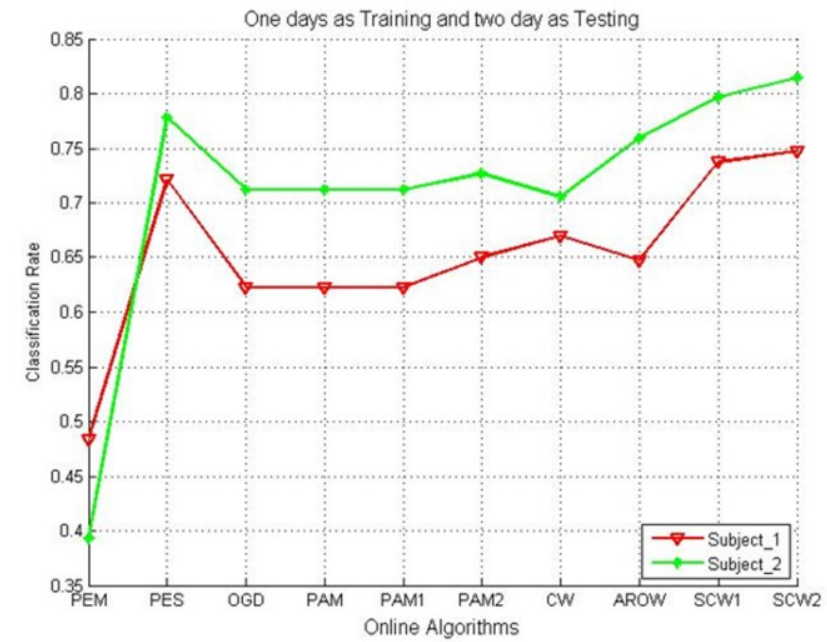

(a)

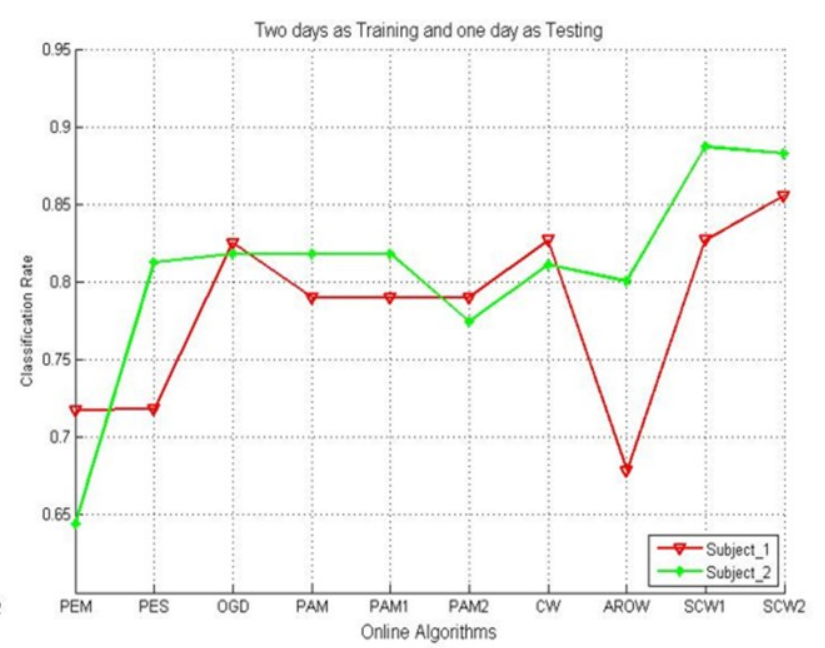

(b)

Fig. 5. Average prediction accuracy of two experiments. (a) Choose one dataset from one session as training data and the other two session as testing data, three experiments will be carried out. (b) Choose two datasets from any two different sessions in any permutation and the left session as testing dataset, six experiments will be implemented.

cross validation. Three sessions of each subject will be run separately in all online learning algorithms, then the average of the cumulative mistakes rate from sessions will be count in each algorithms, the result can be seen in figure 4 . The tendency is the same with the above result. It further uncovered the steady of the second-order algorithms is better than firstorder in cumulative mistake rate.

\section{B. Inter-session performance of the online algorithms}

Expect to reveal the intrinsic characteristic of online learning algorithms. Some exploration in order to improve the robust prosthetic control in long-term use will be carried out between different sessions. By assigning the number and sequence of the sessions into the training data and testing data, two types of experiment will be conducted. Firstly, only one session would be selected to be a training data and 10 random permutation method will be used in training data to generate 10 different training groups, the other two act as the testing data. The experiment would be run in separately by each subject. The result will be seen in Fig. 5 (a). As is seen, the classification rate of SCW2 is up to $78 \%$ and the perceptron with max-score is the worst. In addition, the tendency of curve between two subjects is consistent which prove the performance of classification is steady between subjects. The second experiment would add the number of training data to two. Because the instance used for training in online learning algorithm is executed one by one. So the sequence of sessions in training data will be considered. The result is seen in Fig. 6 (b). The best classification rate is up to $86 \%$ and SCW2 performs best in this experiment.

The classification rate of two experiments is list in table 1. The accuracy is prominently lifted after adding a session to training data. Apart from the improved performance in prediction accuracy, online learning algorithms can gain the new training instance gradually which reduce the unnecessary time spent in retraining like batch algorithm. Once user want to update model with new training data, the operation will execute based on previous model.
TABLE I. THE LIFTING PREDICTION ACCURACY AFTER ADDING THE NUMBER OF TRAINING FROM ONE TO TWO.

\begin{tabular}{|l|c|c|c|c|c|c|}
\hline & \multicolumn{2}{|c|}{ Experiment 1 } & \multicolumn{2}{c|}{ Experiment 2 } & \multicolumn{2}{c|}{ Lifting } \\
\cline { 2 - 7 } & Sub. 1 & Sub. 2 & Sub. 1 & Sub. 2 & Sub. 1 & Sub. 2 \\
\hline PEM & 48.4 & 39.3 & 71.8 & 64.4 & 23.4 & 25.1 \\
\hline PES & 72.1 & 77.8 & 71.9 & 81.3 & -0.2 & 3.5 \\
\hline OGD & 62.3 & 71.2 & 82.5 & 81.8 & 20.2 & 10.6 \\
\hline PAM & 62.3 & 71.2 & 79.0 & 81.8 & 16.7 & 10.6 \\
\hline PAM1 & 62.3 & 71.2 & 79.0 & 81.8 & 16.7 & 10.6 \\
\hline PAM2 & 65.1 & 72.7 & 79.0 & 77.4 & 13.9 & 4.7 \\
\hline CW & 66.9 & 70.6 & 82.7 & 81.1 & 15.8 & 10.5 \\
\hline AROW & 64.7 & 76.0 & 67.7 & 80.0 & 3.0 & 4.0 \\
\hline SCW1 & 73.8 & 79.7 & 82.7 & 88.7 & 8.9 & 9.0 \\
\hline SCW2 & 74.71 & 81.47 & 85.54 & 88.28 & 10.8 & 6.8 \\
\hline
\end{tabular}

\section{Discussion.}

The results show that SCW has an excellent performance on both classify the labelled training data and predict the unlabeled instance. In addition, The SCW is not a timeconsuming algorithm in each learning step which can guarantee the real-time processing. $\mathrm{CW}$ and AROW are good in classification but failed in prediction.

Surface myoelectric signal is nonstationary [25] and easy to be contaminated by a wide variety of factors. It always bring different kind of noise and lead to the changes in EMG patterns for prosthetic control. So the classification method should be capable of handling noise. Meanwhile, easy to retraining in long-term is also essential in prosthetic hand control.

CW learning uses aggressiveness scheme to yield a rapid learning process which borrowed form PA algorithm. It would try it best to adjust the weight to make the new instance be classified to special label, but label noise in new instance will degrade performance in re-training process. So AROW was proposed to compensate for the drawback of $\mathrm{CW}$ in dealing with noise label by introducing adaptive regularization of the prediction function, but discarding the property of adaptive margin make it perform badly. The SCW algorithm is proposed based on the CW learning and AROW learning algorithms. It 
owns many properties, e.g. large margin, confidence, nonseparable and adaptive margin.

Large margin classification is proved to be effective in multiclass classification [32]. In addition, confidence weight and adaptive margin are effective in our study by comparing the different performance among $\mathrm{CW}$, AROW and SCW. The second-order algorithms outperform the first-order suggest us the importance of the distribution of weight vector.

Also, all online learning algorithms can update the existing model continuously whenever a new training sample come up, which is good for general batch algorithms in continuously training. The characteristic of online learning reveals that online learning with adaptive margin and confidence weight are suitable to process this kind of signal in noise environment and long-term use.

\section{CONCLUSION}

In this paper, six popular online learning algorithms are discussed for prosthetic hand control. Three groups of experiment was designed to explore the possibility that classification accuracy on surface myoelectric signal. The experiment result show that the soft confidence weight learning algorithm is the optimal method in different measurement. Further, the characteristic of SCW reveals that a classifier with properties of large margin, confidence weight and adaptive margin can perform good classification property in classification problems.

\section{REFERENCES}

[1] H. Daley, K. Englehart, L. Hargrove, and U. Kuruganti, "High density electromyography data of normally limbed and transradial amputee subjects for multifunction prosthetic control," J. Electromyogr. Kinesiol., vol. 22, no. 3, pp. 478-484, 2012.

[2] K. Englehart and B. Hudgins, "A robust, real-time control scheme for multifunction myoelectric control," IEEE Trans Biomed Eng., vol. 50, pp. 848-54, Jul. 2003

[3] E. Scheme and K. Englehart, "Electromyogram pattern recognition for control of powered upper-limb prostheses: State of the art and challenges for clinical use," J. Rehabil. Res. Development, vol. 48, no. 6 pp. 643-659, 2011.

[4] N. Jiang, S. Dosen, K.-R. Muller, and D. Farina. (2012) Myoelectric Control of Artificial Limbs - Is There a Need to Change Focus? IEEE Signal Processing Magazine. 148-152.

[5] D. Tkach, H. Huang, and T. Kuiken, "Study of stability of time-domain features for electromyographic pattern recognition," J. Neuroeng. Rehabil., vol. 7:21, p. 13, 2010.

[6] L. Hargrove, K. Englehart, and B. Hudgins, "A training strategy to reduce classification degradation due to electrode displacements in pattern recognition based myoelectric control," Biomed. Signal Process. Control, vol. 3, pp. 175-180, 2008.

[7] G. Y., P. Zhou, and G. Li, "Toward attenuating the impact of arm positions on electromyography pattern-recognition based motion classification in transradial amputees," J. NeuroEng. Rehab., vol. 9, no. 74,2012,Available:http://www.jneuroengrehab.com/content/9/1/74/abstr act.

[8] M. Powell, R. Kaliki, and N. Thakor, "User training for pattern recognition-based myoelectric prostheses: Improving phantom limb movement consistency and distinguishability," IEEE Trans. Neural Syst. Rehabil. Eng., vol. 22, no. 3, pp. 522-532, May 2014.

[9] L. Hargrove, K. Englehart, and B. Hudgins, "The effect of electrode displacements on pattern recognition based myoelectric control," in Proc. 28th IEEE Eng. Med. Biol. Soc. Annu. Int. Conf., New York, 2006, pp. 2203-2206.

[10] J. Liu, "Adaptive myoelectric pattern recognition toward improved multifunctional prosthesis control," Med Eng Phys, vol. 37, pp. 424-30, Apr 2015.

[11] X. Chen et al., "Application of a self-enhancing classification method to electromyography pattern recognition for multifunctional prosthesis control," J. Neuroeng. Rehabil., vol. 10, no. 1, p. 44, 2013.

[12] D. Nishikawa, W. W. Yu, H. Yokoi, and Y. Kakazu, "On-line supervising mechanism for learning data in surface electromyogram motion classi- fiers," Syst. Comput. Jpn., vol. 33, pp. 1-11, 2002.

[13] F. Rosenblatt. "The perceptron: A probabilistic model for information storage and organization in the brain”. Psych. Rev., vol.65,pp:386-408, 1958

[14] K. Crammer and Y. Singer, "Ultraconservative Online Algorithms for Multiclass Problems," School Comput. Sci. Eng., Hebrew Univ., Tech. Rep., 2001.

[15] N. Cesa-Bianchi, A. Conconi, and C. Gentile, "A second-order perceptron algorithm," SIAM J. Comput., vol. 34, pp. 640-688, 2005.

[16] K. Crammar, O. Dekel, J. Keshet, S. Shalev-Shawartz, and Y. Singer, "Online passive-aggressive algorithms," J. Mach. Learn. Res., vol. 7, pp. 551-585, Mar. 2006.

[17] Y. Freund and R. Schapire. Large margin classification using the perceptron algorithm. Machine Learning, 37:277-296. 1999.

[18] K. Crammer, M. Dredze, and F. Pereira, "Exact convex confidence weighted learning," in NIPS, Dec. 2008, pp. 345-352.

[19] K. Crammer, A. Kulesza, and M. Dredze, "Adaptive regularization of weight vectors," in NIPS, 2009, pp. 414-422.

[20] C. Castellini and P. van der Smagt, "Surface EMG in advanced hand prosthetics," Biol. Cybern., vol. 100, pp. 35-47, 2009.

[21] K. Crammer, M. Dredze, and A. Kulesza, "Multi-class confidence weighted algorithms," in Proc. 2009 Conf. EMNLP, pp. 496-504.

[22] J. Wang, P. Zhao, and S. C. H. Hoi, "Exact soft confidenceweighted learning," in Proc. 29th ICML, Edinburgh, U.K., 2012.

[23] M. Zinkevich, "Online convex programming and generalized infinitesimal gradient ascent," in Proceedings of the International Conference on Machine Learning, 2003.

[24] Y. Fang, and H. H. Liu, "Robust sEMG Electrodes Configuration for Pattern Recognition based Prosthesis Control," in 2014 International Conference, ICSMC 2014, pp. 2210-2215.

[25] J. Rafiee, M. A. Rafiee, F. Yavari. and M. P. Schoen. "Featrure extraction of foream emg signals for prosthetics." Expert System with applications, vol. 38. no. 4. 4058-4067,2011.

[26] Y. Fang, X. Y. Zhu, and H. H. Liu, "Development of a surface emg acquisition system with novel electrodes configuration and signal representation," in 6th International Conference, ICIRA 2013, pp. 405 414.

[27] M. A. Oskoei and H. Hu, "Myoelectric control systems-a survey," Biomed. Signal Process. Control (Elsevier), vol. 2, no. 4, pp. 275-294, Oct. 2007.

[28] A. Phinyomark, F. Quaine, S. Charbonnier, C. Serviere, F TarpinBernard, and Y. Laurillau, "EMG feature evaluation for improving myoelectric pattern recognition robustness," Expert Syst. Appl., vol. 40, no. 12, pp. 4832-4840, 2013.

[29] B. S. Hudgins, P. A. Parker, and R. N. Scott, "A new strategy for multifunction myoelectric control," IEEE Trans. Biomed. Eng., vol. 40, pp. 82-94, Jan. 1993.

[30] A. D. C. Chan and K. Englehart, "Continuous myoelectric control for powered prostheses using hidden markov models," IEEE Trans. Biomed. Eng., vol. 52, no. 1, pp. 121-124, Jan. 2005.

[31] J. Chu, I. Moon, and M. Mun, "A real-time EMG pattern recognition system based on linear-nonlinear feature projection for a multifunction myoelectric hand," IEEE Trans. Biomed. Eng., vol. 53, no. 11, pp. 2232-2239, Nov. 2006

[32] I. Tscochantaridis, T. Joachims, T. Hofmann, Y. Alun, "Large margin methods for structured and interdependent output variables", J. Mach Learn. Res., vol. 6, pp. 1453-1484, Sep. 2005 